\title{
Multistage gedrite in gedrite-hypersthene-bearing high-grade granulites from Daltonganj, Chhotanagpur granite-gneissic complex, Jharkhand, as evident from TEM and textural relations
}

\author{
S B Dwivedi*, Ravi Ranjan Kumar and Manish Srivastava \\ Department of Civil Engineering, Indian Institute of Technology (BHU), Varanasi 221 005, India. \\ *Corresponding author.e-mail: sbd.civ@itbhu.ac.in
}

MS received 22 December 2017; revised 20 January 2018; accepted 12 May 2018; published online 6 February 2019

The studied rock consists of mineral phases garnet-cordierite-gedrite-hypersthene-biotite-quartz and lies nearly $14 \mathrm{~km}$ southwest of Daltonganj in the western part of Chhotanagpur granite-gneissic complex. Textural relations of mineral phases, mineral chemistry and transmission electron microscopy (TEM) analyses of the rock samples suggest the early (gedrite1), middle (gedrite2) and late stages (gedrite3) formation of gedrite. Hypersthene appears through the metamorphic reaction gedrite + quartz $=$ orthopyroxene + garnet + cordierite $+\mathrm{H}_{2} \mathrm{O}$ due to the breakdown of gedrite 3 at the thermal peak of $869^{\circ} \mathrm{C} / 7.79 \mathrm{kbar}$. The bundles, prismatic and fibrous forms of three gedrites have been observed in the TEM images. TEM images and selected area electron diffraction patterns show the distribution of metallic element position at the different lattice site. The $P-T$ estimates of the rock from garnet-orthopyroxene, garnet-cordierite and garnet-biotite exchange geothermometers and garnet-sillimanite-cordierite-quartz geobarometers vary from $775^{\circ}$ to $869^{\circ} \mathrm{C}, 642^{\circ}$ to $703^{\circ} \mathrm{C}, 480^{\circ}$ to $617^{\circ} \mathrm{C}$ and 6.76 to $7.79 \mathrm{kbar}$, respectively.

Keywords. Hypersthene-bearing gneiss; thermal peak; TEM; metamorphism and $P-T$ condition.

\section{Introduction}

Gedrite-bearing assemblages have been reported from regionally metamorphosed amphibolite to granulite facies rocks with diverse mineral parageneses from different geological areas (Robinson et al. 1982; Baker et al. 1987; Spear 1993; Dasgupta et al. 1999 and references therein and Peck and Smith 2005). The assemblages containing gedrite and hypersthene are more significant in highgrade granulite facies rocks because it preserves the textural evidence of the appearance of gedrite and hypersthene in the rocks. The occurrence of gedrite-bearing rocks in regional metamorphism from different granulite belts of India has attracted the attention of petrologists (The Eastern Ghats, Dasgupta et al. 1999; Southern Granulite, Lal et al. 1984; Rajasthan Granulite, Sharma and Mac Rae 1981). Cordierite- and gedrite-bearing rocks have attracted considerable attention among petrologists due to their unusual bulk composition and the complex texture they have preserved during their formation (Arnold and Sandiford 1990 and references therein). Their interpretation has wide application for the distribution and behaviour of fluids in metamorphic evolution. The occurrence of multistage gedrite phases, in particular assemblage, serves a better tool to understand 
the evolution of the rocks during regional metamorphism. The purpose of this paper is first to report the occurrences of gedrite-hypersthenebearing mineral parageneses from southwest of Daltonganj with their detailed petrography, mineral chemistry and metamorphic evolution.

\subsection{Geological setting of the area under investigation}

The studied area (latitude $23^{\circ} 54^{\prime} 50^{\prime \prime}-23^{\circ} 58^{\prime} 30^{\prime \prime} \mathrm{N}$ and longitude $84^{\circ} 2^{\prime}-84^{\circ} 06^{\prime} 30^{\prime \prime} \mathrm{E}$ ) belongs to the western part of Chhotanagpur granite-gneiss complex (CGGC) within Hazaribagh-Giridih belt. The CGGC covers an area of about $80,000 \mathrm{~km}^{2}$ and extends west to east across the states of Madhya Pradesh, Jharkhand and West Bengal (Ghose 1983, 1992). The area lies nearly $14 \mathrm{~km}$ southwest of Daltonganj and consists of the high-grade regionally metamorphosed metapelites. This gneissic complex is of composite character consisting mainly of granitoid gneisses, migmatites and massive granite with enclaves of metasedimentary and meta-igneous rocks and intrusive of basic and intermediate rock types (Ghose 1983, 1992; Chatterjee and Ghose 2011; Yadav et al. 2016 and reference therein). The gedrite-hypersthene-bearing gneiss occurs as enclaves within CGGC.

The study area comprises a variety of rocks, viz., basic granulite, charnockite, gedrite-garnetbiotite-gneiss, garnet-cordierite-sillimanite gneiss, sillimanite-graphite-schist, amphibolite, dolerite in the north-western extension of the CGGC in the localities Sokra, Datam and MahawatMuria in the SW direction of the Daltonganj (figure 1).

In the study area, general strike is found to be NW-SE to N-S to NE-SW direction. But, on region scale, a general $\mathrm{E}-\mathrm{W}$ strike with northerly dip (less commonly southerly) of the dominant foliation is present in the CGGC (Sengupta and Sarkar 1964, 1968; Ghose 1983; Mazumdar 1988; Sarkar 1988; Baidya et al. 1989; Srivastava and Ghose 1992; Goswami and Bhattacharyya 2010; Karmakar et al. 2011).

\section{Petrography and mineral chemistry}

\subsection{Megascopic characters}

The garnet-cordierite-gedrite and garnetcordierite-gedrite-hypersthene gneisses are generally fresh having resinous and greasy appearance. They are medium to coarse grained having garnet as one of their main constituent minerals displaying pinkish colour. The cordierite bands have grey to light reddish brown colour. The presence of garnet in abundance in these rocks has imparted a reddish tinge to the rock. In these gneisses coarse biotite flakes are present. Grey to black colour of very coarse-grained gedrite can be easily observed in the hand-specimen (figure 2a).

\subsection{Microscopic characters}

Microscopic studies of the gneisses (garnetcordierite-gedrite gneiss and garnet-cordieritegedrite-hypersthene gneiss) show the three distinct types of mineral assemblages in which either hypersthene is present or absent in the following paragenesis:

(i) garnet-gedrite-cordierite-chlorite-biotitequartz,

(ii) garnet-cordierite-gedrite-biotite-albitequartz,

(iii) garnet-cordierite-gedrite-hypersthenebiotite-quartz.

The mineral abbreviations used in this study are from Whitney and Evans (2010).

Chlorite occurs in the form of medium to coarse flakes and with an association of biotite or also as individual laths. It occurs as inclusion within gedrite and consumed to form garnet 1 and gedrite1 due to the reaction (figure $2 \mathrm{~b}$ )

$$
\text { chlorite }+ \text { quartz }=\text { garnet } 1+\text { gedrite } 1+\mathrm{H}_{2} \mathrm{O} .
$$

Biotite shows the characteristic pleochroism, $\mathrm{X}=$ straw yellow, $\mathrm{Y}=\mathrm{Z}=$ dark brown, where $\mathrm{X}<\mathrm{Y}=\mathrm{Z}$. It occurs in the form of coarse flakes and as individual laths wrapping around garnet, cordierite, gedrite. Majority of the biotite grains are associated with chlorite and chlorite appears similar to biotite. The inclusion of trail of Al-rich biotite in gedrite grain (figure 2c), which is in contact with cordierite, suggests the reaction

Al-rich biotite + quartz $=$ gedrite $1+$ cordierite

$$
+\mathrm{K} \text {-feldspar }+\mathrm{H}_{2} \mathrm{O} \text {. }
$$

Garnet occurs as coarse xenoblast and poikiloblast and containing biotite, gedrite, cordierite and quartz as inclusion (figure 2d). Garnet also contains other heavy minerals as inclusion, i.e., zircon, monazite, ilmenite, magnetite, etc. Some of the 


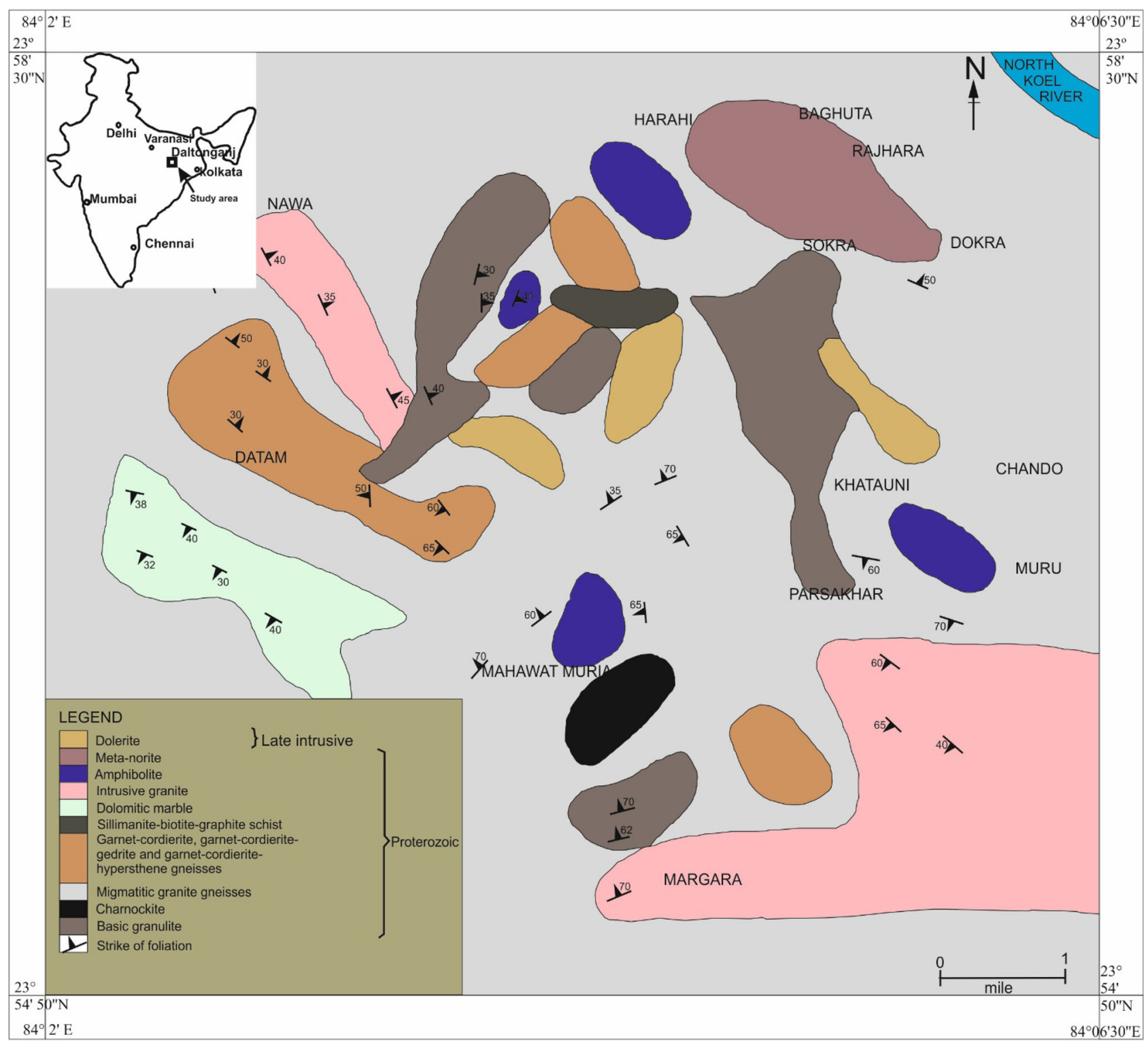

Figure 1. Geological map of the area around southwest of Daltonganj, District Palamau, Bihar.

garnet grains are completely rimmed by cordierite and the further rimmed by gedrite (figure 2e). This corona texture predicts the reaction

garnet $1+$ quartz $+\mathrm{H}_{2} \mathrm{O}=$ cordierite + gedrite 2 .

Garnet and quartz at places were completely wrapped by gedrite coexisting with cordierite (figure 2f). This textural relation suggests the reaction

garnet $1+$ quartz $=$ gedrite $2+$ cordierite $+\mathrm{H}_{2} \mathrm{O}$.

A corona texture is observed in which corroded garnet and boitite are partially wrapped by cordierite and gedrite (figure $2 \mathrm{~g}$ ) provides the evidence of reaction

garnet $1+$ biotite + quartz $=$ gedrite $2+$ cordierite

$$
+\mathrm{K} \text {-feldspar }+\mathrm{H}_{2} \mathrm{O} \text {. }
$$

Garnet shows the two distinct stages as garnet1 which is the dominant garnet in the rocks coexisting with gedrite, cordierite and biotite.

Cordierite occurs as coarse xenoblast or granular aggregates rimming xenoblast of garnet and gedrite (figure $2 \mathrm{~b}-\mathrm{h}$ ). At some places cordierite rims the xenoblasts of garnet (figure $2 \mathrm{~g}$ ) and biotite flakes. The textural relation suggests that the cordierite is formed due to the breakdown of garnet in the presence of biotite and quartz by reaction (5). Cordierite shows alteration along fractures and grain boundaries. 

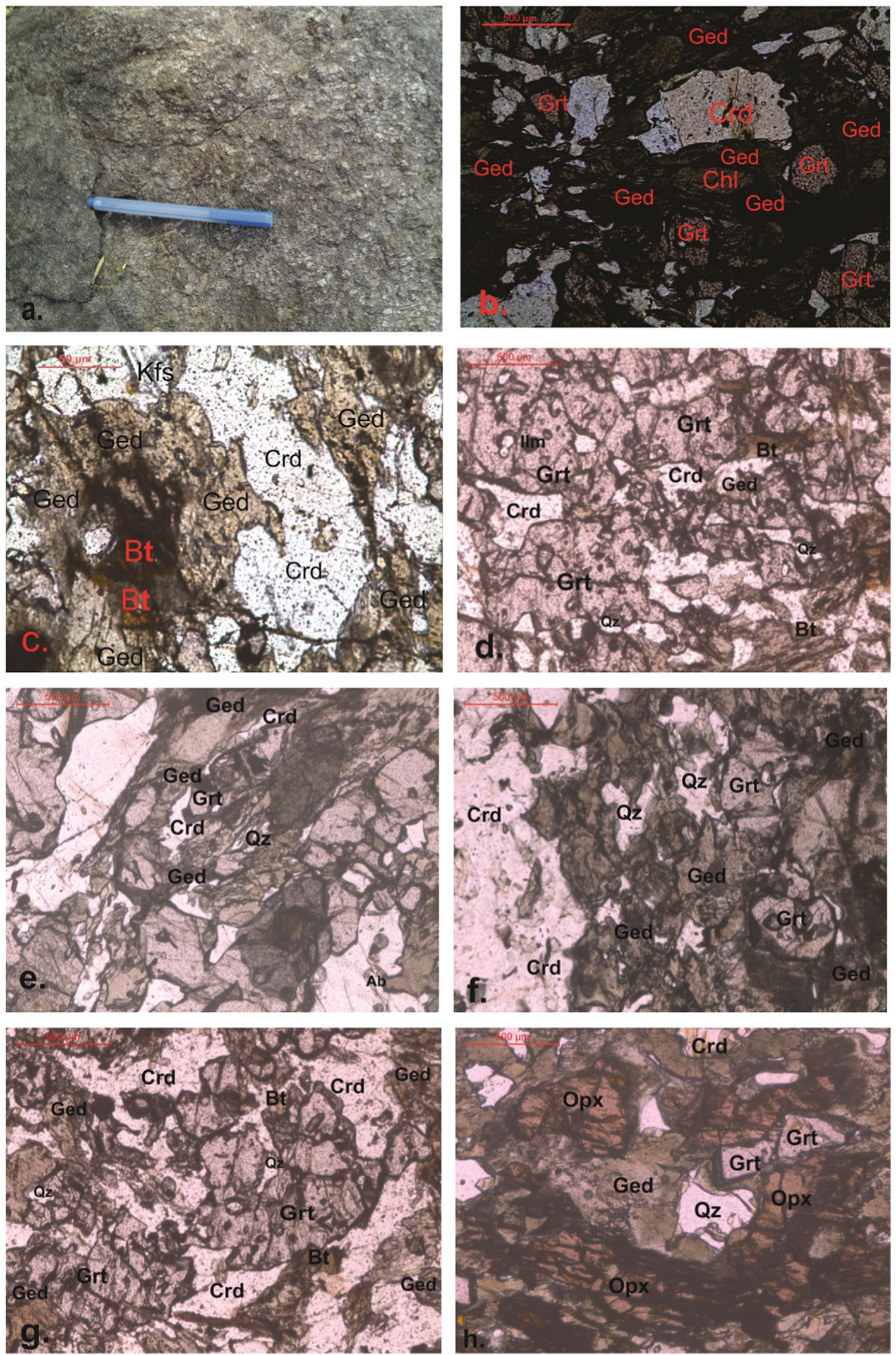

Figure 2. (a) Field photograph of garnet-cordierite-orthopyroxene-gedrite gneiss; photomicrographs illustrating the textural relations in garnet-cordierite-orthopyroxene-gedrite gneiss. (b) Chlorite completely rimmed by gedrite. (c) Al-rich biotite partially rimmed by gedrite. (d) Inclusion of cordierite, gedrite, biotite, quartz and ilemanite in garnet. (e) Corona texture in which garnet is rimmed by cordierite followed by gedrite. (f) Garnet rimmed by gedrite. (g) Garnet and biotite surrounded by cordierite and gedrite. (h) Gedrite rimmed by orthopyroxene and garnet occurs as inclusion within orthopyroxene. 
Gedrite is coarse grained, idioblastic, prism and commonly associated with biotite to define foliation in the rocks. It is pleochroic, $\mathrm{X}=$ pale yellowish green, $\mathrm{Z}=$ dark greenish brown, $\mathrm{X}>\mathrm{Y}>\mathrm{Z}$. The gedrite shows parallel extinction and in this rock, we observed three morphological types of gedrite crystals. The first one is the elongated fibrous grain $(2-3 \mathrm{~mm})$ and the thickness is $0.15-0.20 \mathrm{~mm}$, and forms foliation in the rocks (figure $2 \mathrm{~b}$ ). The second type of grain occurs as idioblastic, this type of grain occurs in huge amount in this rock (figure $2 \mathrm{c}-\mathrm{e}$ ) and third one is prismatic in nature (figure $2 \mathrm{~h}$ ). Therefore, three types of gedrite1, gedrite 2 and gedrite 3 formed at different times during the crystallisation process. Gedrite1 is formed after the consumption of chlorite and biotite from reactions (1 and 2) and contains the inclusion of chlorite and biotite (figure $2 \mathrm{~b}$ and $\mathrm{c}$ ). Gedrite2 contains the inclusion of garnet1 and cordierite and forms due to reaction (3-5). Gedrite3 is corroded, prismatic and occurs as inclusion in orthopyroxene.

Orthopyroxene is medium to coarse-grained (1$3 \mathrm{~mm}$ ) and idioblastic to xenoblastic in nature. It is reddish pink in colour due to iron enrichment with strong pleochroism $\mathrm{X}$ - yellow, $\mathrm{Y}$ - pink, Z green; $\mathrm{X}<\mathrm{Y}<\mathrm{Z}$. At places, gedrite + quartz are completely rimmed by orthopyroxene, garnet and cordierite (figure $2 \mathrm{~h}$ ). This textural relation provides the evidence through which hypersthenes and garnet2 appear in the rocks due to the reaction

$$
\begin{aligned}
\text { gedrite } 3+\text { quartz }= & \text { orthopyroxene }+ \text { garnet } 2 \\
& + \text { cordierite }+\mathrm{H}_{2} \mathrm{O} .
\end{aligned}
$$

The second state of the garnet named as garnet2 coexists with orthopyroxene and formed due to breakdown of gedrite.

\subsection{Mineral chemistry}

The analytical work was carried out on the electron probe microanalysis (EPMA) CAMECA SX Five instrument at DST-SERB National Facility, Department of Geology, Centre of Advanced Study, Institute of Science, Banaras Hindu University. Polished thin section was coated with $20 \mathrm{~nm}$ thin layer of carbon for electron probe microanalyses using LEICA-EM ACE200 instrument. The CAMECA SX Five instrument was operated at a voltage of $15 \mathrm{kV}$ and current $10 \mathrm{nA}$ with a LaB6 source in the electron gun for generation of the electron beam. Natural silicate mineral andradite as an internal standard was used to verify positions of crystals (SP1-TAP, SP2-LiF, SP3-LPET, SP4-TAP and SP5-PC1) concerning the corresponding wavelength dispersive (WD) spectrometers (SP\#) in CAMECA SX Five instrument. The following $\mathrm{X}$-ray lines were used in the analyses: $\mathrm{F}-\mathrm{K} \alpha, \mathrm{Na}-\mathrm{K} \alpha, \mathrm{Mg}-\mathrm{K} \alpha, \mathrm{Al}-\mathrm{K} \alpha, \mathrm{Si}-\mathrm{K} \alpha$, $\mathrm{P}-\mathrm{K} \alpha, \mathrm{K}-\mathrm{K} \alpha, \mathrm{Cl}-\mathrm{K} \alpha, \mathrm{Ca}-\mathrm{K} \alpha$, Ti-K $\alpha, \mathrm{Cr}-\mathrm{K} \alpha, \mathrm{Mn}-$ $\mathrm{K} \alpha, \mathrm{Fe}-\mathrm{K} \alpha, \mathrm{Ni}-\mathrm{K} \alpha$ and Sr-L $\alpha$. Natural mineral standards: apatite, albite, halite, periclase, peridotite, corundum, wollastonite, orthoclase, rutile, chromite, rhodonite, celestite, barite, hematite and synthetic $\mathrm{Ni}$ metal supplied by CAMECAAMETEK were used for routine calibration and quantification. Routine calibration, acquisition, quantification and data processing were carried out using SxSAB version 6.1 and SX-Results software of CAMECA. The electron microprobe analysis of different minerals is presented in tables $1-6$.

Table 1. Electron probe microanalysis (EPMA) and structural formula of orthopyroxene, from the garnet-cordierite-

\begin{tabular}{|c|c|c|c|c|c|}
\hline \multirow[b]{2}{*}{ Domain } & \multicolumn{5}{|c|}{ Sample R-91-97 } \\
\hline & $71 / 1$ & $73 / 1$ & $75 / 1$ & $76 / 1$ & $79 / 1$ \\
\hline $\mathrm{SiO}_{2}$ & 49.14 & 48.79 & 48.93 & 49.07 & 48.63 \\
\hline $\mathrm{TiO}_{2}$ & 0.00 & 0.00 & 0.00 & 0.00 & 0.00 \\
\hline $\mathrm{Al}_{2} \mathrm{O}_{3}$ & 2.89 & 2.91 & 2.97 & 2.72 & 3.13 \\
\hline $\mathrm{Cr}_{2} \mathrm{O}_{3}$ & 0.00 & 0.00 & 0.00 & 0.00 & 0.00 \\
\hline $\mathrm{FeO}$ & 28.78 & 28.45 & 28.70 & 30.32 & 28.54 \\
\hline $\mathrm{MnO}$ & 0.00 & 0.00 & 0.00 & 0.00 & 0.00 \\
\hline $\mathrm{MgO}$ & 17.94 & 17.97 & 17.53 & 17.75 & 17.94 \\
\hline $\mathrm{CaO}$ & 0.02 & 0.05 & 0.03 & 0.00 & 0.02 \\
\hline $\mathrm{Na}_{2} \mathrm{O}$ & 0.00 & 0.01 & 0.01 & 0.02 & 0.06 \\
\hline $\mathrm{K}_{2} \mathrm{O}$ & 0.00 & 0.00 & 0.00 & 0.00 & 0.00 \\
\hline Total & 99.21 & 98.41 & 98.54 & 100.04 & 98.38 \\
\hline \multicolumn{6}{|l|}{6 oxygen } \\
\hline $\mathrm{Si}$ & 1.91 & 1.91 & 1.91 & 1.90 & 1.90 \\
\hline $\mathrm{Al}^{\mathrm{IV}}$ & 0.09 & 0.09 & 0.08 & 0.10 & 0.10 \\
\hline$\Sigma \mathrm{Z}$ & 2 & 2 & 2 & 2 & 2 \\
\hline $\mathrm{Al}^{\mathrm{VI}}$ & 0.04 & 0.04 & 0.05 & 0.03 & 0.04 \\
\hline $\mathrm{Fe}^{3+}$ & 0.07 & 0.07 & 0.05 & 0.11 & 0.09 \\
\hline $\mathrm{Cr}$ & 0 & 0 & 0 & 0 & 0 \\
\hline $\mathrm{Ti}$ & 0 & 0 & 0 & 0 & 0 \\
\hline $\mathrm{Fe}^{2+}$ & 0.86 & 0.85 & 0.89 & 0.87 & 0.84 \\
\hline $\mathrm{Mn}$ & 0 & 0 & 0 & 0 & 0 \\
\hline $\mathrm{Mg}$ & 1.04 & 1.05 & 1.02 & 1.03 & 1.05 \\
\hline $\mathrm{Ca}$ & 0.0 & 0.00 & 0.00 & 0 & 0.00 \\
\hline $\mathrm{Na}$ & 0 & 0.00 & 0.00 & 0.00 & 0.01 \\
\hline $\mathrm{K}$ & 0 & 0 & 0 & 0 & 0 \\
\hline$\Sigma \mathrm{Y}$ & 2.02 & 2.02 & 2.01 & 2.04 & 2.02 \\
\hline $\mathrm{X}_{\mathrm{Mg}}$ & 0.53 & 0.53 & 0.52 & 0.51 & 0.53 \\
\hline
\end{tabular}
hypersthene-gedrite gneiss of the study area.

$\mathrm{X}_{\mathrm{Mg}}=\mathrm{Mg} /(\mathrm{Mg}+\mathrm{Fe})$. 
The microprobe analyses data of pyroxenes are plotted in the $\mathrm{CaSiO}_{3}-\mathrm{MgSiO}_{3}-\mathrm{FeSiO}_{3}$ endmember diagram (figure 3 ). The plots of the orthopyroxenes from the granulites of the study area lie close to the hypersthene composition on enstatiteferrosilite line. The $\mathrm{X}_{\mathrm{Mg}}$ orthopyroxene varies between 0.51 and 0.53 (table 1 ).

Garnets show the solid solution dominantly between almandine (65.9-77.1 mol\%), pyrope (20.9-32.0 mol\%), with minor amounts of grossular (0.7-1.7 mol\%) and spessartine (0.3-1.0 mol\%) (figure 4). Garnet1 contains low pyrope which varies from 20.9 to $26.4 \mathrm{~mol} \%$ and garnet2 contains the pyrope which ranges from 31.1 to 32.0 $\mathrm{mol} \%$.

Assignments and stoichiometric constraints of amphiboles are according to the International Mineral Association (IMA) recommendations (Leake

Table 2. EPMA and structural formula of garnet, from the garnet-cordierite-hypersthene-gedrite gneiss of the study area.

\begin{tabular}{|c|c|c|c|c|c|}
\hline \multirow[b]{2}{*}{ Domain } & \multicolumn{5}{|c|}{ Sample R-91-97 } \\
\hline & $122 / 142$ & $122 / 143$ & $69 / 1$ & $70 / 1$ & $123 / 1$ \\
\hline $\mathrm{SiO}_{2}$ & 37.45 & 38.22 & 37.82 & 37.73 & 37.34 \\
\hline $\mathrm{TiO}_{2}$ & 0.05 & 0 & 0 & 0 & 0.01 \\
\hline $\mathrm{Al}_{2} \mathrm{O}_{3}$ & 20.51 & 20.68 & 20.49 & 20.61 & 20.29 \\
\hline $\mathrm{Cr}_{2} \mathrm{O}_{3}$ & 0 & 0 & 0 & 0 & 0 \\
\hline $\mathrm{FeO}$ & 35.13 & 35.79 & 31.66 & 31.58 & 34.67 \\
\hline $\mathrm{MnO}$ & 0.36 & 0.38 & 0.36 & 0.23 & 0.2 \\
\hline $\mathrm{MgO}$ & 5.8 & 5.34 & 8.1 & 7.89 & 6.6 \\
\hline $\mathrm{CaO}$ & 0.27 & 0.41 & 0.51 & 0.58 & 0.26 \\
\hline Total & 99.88 & 100.93 & 99.14 & 98.82 & 99.38 \\
\hline \multicolumn{6}{|l|}{12 oxygen } \\
\hline $\mathrm{Si}$ & 2.99 & 3.02 & 2.98 & 2.99 & 2.97 \\
\hline $\mathrm{Al}^{\mathrm{IV}}$ & 0 & 0 & 0 & 0 & 0 \\
\hline$\Sigma \mathrm{Z}$ & 2.99 & 3.02 & 2.98 & 2.99 & 2.97 \\
\hline $\mathrm{Al}^{\mathrm{VI}}$ & 1.93 & 1.93 & 1.91 & 1.92 & 1.9 \\
\hline $\mathrm{Ti}$ & 0 & 0 & 0 & 0 & 0 \\
\hline $\mathrm{Cr}$ & 0 & 0 & 0 & 0 & 0 \\
\hline $\mathrm{Fe}^{3+}$ & 0.09 & 0.03 & 0.13 & 0.10 & 0.15 \\
\hline$\Sigma Y$ & 2.02 & 1.96 & 2.04 & 2.02 & 2.05 \\
\hline $\mathrm{Fe}^{2+}$ & 2.26 & 2.33 & 1.96 & 1.99 & 2.15 \\
\hline $\mathrm{Mn}$ & 0.02 & 0.03 & 0.02 & 0.02 & 0.01 \\
\hline $\mathrm{Mg}$ & 0.69 & 0.63 & 0.95 & 0.93 & 0.78 \\
\hline $\mathrm{Ca}$ & 0.02 & 0.03 & 0.04 & 0.05 & 0.02 \\
\hline$\Sigma \mathrm{X}$ & 2.99 & 3.02 & 2.97 & 2.99 & 2.96 \\
\hline $\mathrm{X}_{\mathrm{Mg}}$ & 0.23 & 0.21 & 0.33 & 0.32 & 0.26 \\
\hline Pyrope & 23.1 & 20.9 & 32.0 & 31.1 & 26.4 \\
\hline Almandine & 75.5 & 77.1 & 65.9 & 66.5 & 72.6 \\
\hline Grossularite & 0.7 & 1.0 & 1.4 & 1.7 & 0.7 \\
\hline Spessartite & 0.7 & 1.0 & 0.7 & 0.7 & 0.3 \\
\hline
\end{tabular}

$\mathrm{X}_{\mathrm{Mg}}=\mathrm{Mg} /(\mathrm{Mg}+\mathrm{Fe})$.
Table 3. EPMA and structural formula of gedrite, from the garnet-cordierite-hypersthene-gedrite gneiss of the study area.

\begin{tabular}{|c|c|c|c|c|c|}
\hline \multirow[b]{2}{*}{ Domain } & \multicolumn{5}{|c|}{ Sample R-91-97 } \\
\hline & $133 / 1$ & $134 / 1$ & $171 / 1$ & $175 / 1$ & $176 / 1$ \\
\hline $\mathrm{SiO}_{2}$ & 44.49 & 45.15 & 44.7 & 44.43 & 44.91 \\
\hline $\mathrm{TiO}_{2}$ & 0.22 & 0.22 & 0.39 & 0.32 & 0.26 \\
\hline $\mathrm{Al}_{2} \mathrm{O}_{3}$ & 12.3 & 11.36 & 11.65 & 12.01 & 11.83 \\
\hline $\mathrm{FeO}$ & 21.59 & 22.41 & 22.39 & 21.81 & 22.53 \\
\hline $\mathrm{MnO}$ & 0 & 0 & 0 & 0 & 0 \\
\hline $\mathrm{MgO}$ & 15.51 & 15.57 & 14.9 & 14.73 & 14.98 \\
\hline $\mathrm{CaO}$ & 0.09 & 0.1 & 0.13 & 0.11 & 0.09 \\
\hline $\mathrm{Na}_{2} \mathrm{O}$ & 1.5 & 1.47 & 1.6 & 1.67 & 1.61 \\
\hline $\mathrm{K}_{2} \mathrm{O}$ & 0 & 0 & 0 & 0 & 0 \\
\hline $\mathrm{BaO}$ & 0.24 & 0.37 & 0.2 & 0.41 & 0.34 \\
\hline $\mathrm{SrO}$ & 0 & 0 & 0 & 0 & 0 \\
\hline $\mathrm{PbO}$ & 0 & 0 & 0 & 0 & 0 \\
\hline $\mathrm{ZnO}$ & 0 & 0 & 0 & 0 & 0 \\
\hline $\mathrm{F}$ & 0.4 & 0.43 & 0.34 & 0.46 & 0.22 \\
\hline Total & 96.49 & 97.14 & 96.33 & 96.08 & 96.88 \\
\hline \multicolumn{6}{|c|}{23 oxygen } \\
\hline $\mathrm{Si}$ & 6.65 & 6.72 & 6.71 & 6.69 & 6.7 \\
\hline $\mathrm{Al}^{\mathrm{IV}}$ & 1.35 & 1.28 & 1.29 & 1.31 & 1.3 \\
\hline$\Sigma Z$ & 8 & 8 & 8 & 8 & 8 \\
\hline $\mathrm{Al}^{\mathrm{VI}}$ & 0.81 & 0.71 & 0.77 & 0.83 & 0.78 \\
\hline $\mathrm{Ti}$ & 0.02 & 0.02 & 0.04 & 0.04 & 0.03 \\
\hline $\mathrm{Cr}$ & 0 & 0 & 0 & 0 & 0 \\
\hline $\mathrm{Fe}^{3+}$ & 0.03 & 0.05 & 0 & 0 & 0 \\
\hline $\mathrm{Fe}^{2+}$ & 2.66 & 2.74 & 2.81 & 2.75 & 2.81 \\
\hline $\mathrm{Mn}$ & 0 & 0 & 0 & 0 & 0 \\
\hline $\mathrm{Mg}$ & 3.45 & 3.46 & 3.33 & 3.31 & 3.33 \\
\hline$\Sigma X$ & 6.99 & 6.98 & 6.95 & 6.92 & 6.96 \\
\hline $\mathrm{Ca}$ & 0.01 & 0.02 & 0.02 & 0.02 & 0.02 \\
\hline $\mathrm{Na}$ & 0.43 & 0.42 & 0.47 & 0.49 & 0.47 \\
\hline $\mathrm{K}$ & 0 & 0 & 0 & 0 & 0 \\
\hline $\mathrm{Ba}$ & 0.01 & 0.02 & 0.01 & 0.02 & 0.02 \\
\hline$\Sigma Y$ & 0.46 & 0.46 & 0.5 & 0.53 & 0.5 \\
\hline $\mathrm{F}$ & 0.19 & 0.2 & 0.16 & 0.22 & 0.11 \\
\hline $\mathrm{OH}^{*}$ & 1.81 & 1.8 & 1.84 & 1.78 & 1.89 \\
\hline $\mathrm{X}_{\mathrm{Mg}}$ & 0.56 & 0.55 & 0.54 & 0.55 & 0.54 \\
\hline
\end{tabular}

$\mathrm{X}_{\mathrm{Mg}}=\mathrm{Mg} /(\mathrm{Mg}+\mathrm{Fe})$.

$*$ Calculated values.

et al. 1997). The analysed amphiboles have ( $\mathrm{Ca}+$ $\mathrm{Na})<1.0 ;\left(\mathrm{Mg}, \mathrm{Fe}^{2+}, \mathrm{Mn}, \mathrm{Li}\right) \geq 1.0$ and Li-poor and therefore, these are $\mathrm{Mg}-\mathrm{Fe}-\mathrm{Mn}-\mathrm{Li}$ amphiboles according to Leake et al. (1997) classification. When this analysis is plotted on leaks classification diagram (figure 5) then all the plots fall in the gedrite field. In this granulitic rock, amphibole has fluorine content which varies from 0.22 to $0.46 \mathrm{pfu}$. $\mathrm{Al}^{\mathrm{IV}}$ and $\mathrm{Al}^{\mathrm{VI}}$ both are present in sufficient amount $1.27-1.35$ and $0.71-0.82 \mathrm{pfu}$, respectively. The $\mathrm{X}_{\mathrm{Mg}}$ ranges from 0.54 to 0.56 . 
Table 4. EPMA and structural formula of cordierite, from the garnet-cordierite-hypersthene-gedrite gneiss of the study area.

\begin{tabular}{lccccc}
\hline & \multicolumn{5}{c}{ Sample R-91-97 } \\
\cline { 2 - 6 } Domain & $20 / 1(\mathrm{C})$ & $21 / 1(\mathrm{C})$ & $136 / 1(\mathrm{R})$ & $140 / 1(\mathrm{R})$ & $139 / 1(\mathrm{R})$ \\
\hline $\mathrm{SiO}_{2}$ & 49.69 & 49.19 & 48.62 & 48.76 & 48.33 \\
$\mathrm{Al}_{2} \mathrm{O}_{3}$ & 29.82 & 29.24 & 30.62 & 30.38 & 30.38 \\
$\mathrm{FeO}$ & 4.54 & 5.17 & 6.02 & 5.68 & 5.33 \\
$\mathrm{MnO}$ & 0.00 & 0.00 & 0.00 & 0.00 & 0.00 \\
$\mathrm{MgO}$ & 10.61 & 10.61 & 10.16 & 10.30 & 10.17 \\
$\mathrm{Na} 2 \mathrm{O}$ & 0.11 & 0.31 & 0.13 & 0.13 & 0.10 \\
$\mathrm{Total}$ & 94.83 & 94.55 & 95.77 & 95.39 & 94.31 \\
18 oxygen & & & & & \\
$\mathrm{Si}$ & 5.18 & 5.19 & 5.10 & 5.12 & 5.11 \\
$\mathrm{Al}$ & 3.68 & 3.64 & 3.78 & 3.76 & 3.79 \\
$\mathrm{ZZ}$ & 8.86 & 8.8 & 8.88 & 8.88 & 8.90 \\
$\mathrm{Fe}$ & 0.44 & 0.46 & 0.53 & 0.50 & 0.47 \\
$\mathrm{Mn}$ & 0 & 0 & 0 & 0 & 0 \\
$\mathrm{Mg}$ & 1.66 & 1.67 & 1.59 & 1.61 & 1.60 \\
$\Sigma \mathrm{X}$ & 2.10 & 2.13 & 2.12 & 2.1 & 2.08 \\
$\mathrm{Na}$ & 0.02 & 0.06 & 0.03 & 0.03 & 0.02 \\
$\mathrm{X}$ & 0.79 & 0.78 & 0.75 & 0.76 & 0.77 \\
\hline $\mathrm{Xg}$ & & & & & \\
$\mathrm{Mg}$ & $\mathrm{Mg} /(\mathrm{Mg}+\mathrm{Fe}) .(\mathrm{C})$ & $=$ & Core,$(\mathrm{R})=\mathrm{Rim}$. &
\end{tabular}

Biotite contains 1.6-2.08 wt\% $\mathrm{TiO}_{2}$ (table 4). Biotite compositions are magnesian and the $\mathrm{X}_{\mathrm{Mg}}$ $(\mathrm{Mg} / \mathrm{Fe}+\mathrm{Mg})$ of biotite ranges from 0.71 to 0.74 .

Cordierite is magnesian and $\mathrm{X}_{\mathrm{Mg}}$ ranges from 0.75 to 0.79 . In cordierite the presence of $\mathrm{Na}_{2} \mathrm{O}$ is less than $1 \mathrm{wt} \%$ and larger cations are almost absent.

Chlorite contains $\mathrm{Fe}^{2+}$ as well as $\mathrm{Fe}^{3+}$, and in some of the chlorite grains, $\mathrm{Fe}^{3+}$ exceeds than $\mathrm{Fe}^{2+}$. Also, $\mathrm{Al}^{\mathrm{IV}}$ and $\mathrm{Al}^{\mathrm{VI}}$ found in these rocks, which lie between 0 and 0.39 and 4.59 and 5.27 pfu, respectively. $\mathrm{BaO}$ is found in trace amount and $\mathrm{X}_{\mathrm{Mg}}$ varies from 0.37 to 0.45 .

\section{Transmission electron microscopy (TEM) analysis}

TEM was done at the Central Instrumental Facility of the Indian Institute of Technology (BHU) at Varanasi with an FEI-Philips (Type: TECNAI G 20 TWIN) operating at 210-240 V.

\subsection{Gedrite}

The microstructures observed in the rock sample are the results of the petrological conditions. TEM images and selected area electron diffraction (SAED) patterns of the gedrite
Table 5. EPMA and structural formula of biotite, from the garnet-cordierite-hypersthene-gedrite gneiss of the study area.

\begin{tabular}{|c|c|c|c|c|c|}
\hline \multirow[b]{2}{*}{ Domain } & \multicolumn{5}{|c|}{ Sample R-91-97 } \\
\hline & $157 / 1$ & $160 / 1$ & $161 / 1$ & $162 / 1$ & $122 / 146$ \\
\hline $\mathrm{SiO}_{2}$ & 39.73 & 39.22 & 38.27 & 38.77 & 40.42 \\
\hline $\mathrm{TiO}_{2}$ & 1.64 & 1.83 & 2.08 & 1.87 & 1.98 \\
\hline $\mathrm{Al}_{2} \mathrm{O}_{3}$ & 14.07 & 13.71 & 14.38 & 14.61 & 15.38 \\
\hline $\mathrm{FeO}$ & 12.21 & 12.86 & 12.02 & 12.38 & 12.10 \\
\hline $\mathrm{MnO}$ & 0 & 0 & 0 & 0 & 0.00 \\
\hline $\mathrm{MgO}$ & 18.49 & 18.15 & 18.05 & 17.78 & 17.66 \\
\hline $\mathrm{CaO}$ & 0.05 & 0.01 & 0.01 & 0.02 & 0.00 \\
\hline $\mathrm{BaO}$ & 0.21 & 0.10 & 0.21 & 0.10 & 0.42 \\
\hline $\mathrm{Na}_{2} \mathrm{O}$ & 0.72 & 0.56 & 0.59 & 0.65 & 0.71 \\
\hline $\mathrm{K}_{2} \mathrm{O}$ & 9.01 & 9.11 & 8.85 & 8.94 & 8.82 \\
\hline $\mathrm{Cl}$ & 0.09 & 0.04 & 0.04 & 0.06 & 0.05 \\
\hline $\mathrm{F}$ & 1.68 & 2.24 & 2.22 & 2.02 & 0.51 \\
\hline Total & 97.91 & 97.82 & 96.70 & 97.20 & 98.05 \\
\hline \multicolumn{6}{|c|}{22 oxygen } \\
\hline $\mathrm{Si}$ & 5.78 & 5.76 & 5.67 & 5.71 & 5.66 \\
\hline $\mathrm{Al}^{\mathrm{IV}}$ & 2.22 & 2.24 & 2.33 & 2.29 & 2.34 \\
\hline$\Sigma \mathrm{Z}$ & 8 & 8 & 8 & 8 & 8 \\
\hline $\mathrm{Al}^{\mathrm{VI}}$ & 0.19 & 0.14 & 0.19 & 0.24 & 0.28 \\
\hline $\mathrm{Ti}$ & 0.18 & 0.20 & 0.23 & 0.21 & 0.20 \\
\hline $\mathrm{Fe}^{2+}$ & 1.49 & 1.58 & 1.49 & 1.52 & 1.42 \\
\hline $\mathrm{Mn}$ & 0 & 0 & 0 & 0 & 0 \\
\hline $\mathrm{Ba}$ & 0.01 & 0.01 & 0.01 & 0.01 & 0.02 \\
\hline $\mathrm{Mg}$ & 4.01 & 3.98 & 3.99 & 3.90 & 4 \\
\hline$\Sigma X$ & 5.88 & 5.91 & 5.91 & 5.88 & 5.92 \\
\hline $\mathrm{Ca}$ & 0.01 & 0.01 & 0.00 & 0.01 & 0 \\
\hline $\mathrm{Na}$ & 0.20 & 0.16 & 0.17 & 0.19 & 0.19 \\
\hline $\mathrm{K}$ & 1.67 & 1.71 & 1.67 & 1.68 & 1.57 \\
\hline$\Sigma Y$ & 1.88 & 1.87 & 1.84 & 1.87 & 1.77 \\
\hline $\mathrm{Cl}$ & 0.02 & 0.0 & 0.01 & 0.01 & 0.01 \\
\hline $\mathrm{F}$ & 0.77 & 1.04 & 1.04 & 0.94 & 0.22 \\
\hline $\mathrm{X}_{\mathrm{Mg}}$ & 0.73 & 0.72 & 0.73 & 0.72 & 0.74 \\
\hline
\end{tabular}

$\mathrm{X}_{\mathrm{Mg}}=\mathrm{Mg} /(\mathrm{Mg}+\mathrm{Fe})$.

containing sample are shown in figure $6(\mathrm{a}-\mathrm{f})$. Here three types, i.e., bundles, prismatic and fibrous forms of gedrite have been observed in the TEM images (figure 6a and b). Bundles are assemblages of individual separable particles (they are fibres, but also acicular or prismatic particles, figure $6 \mathrm{a}$ ). Bundles often show lack of cohesion between particles in the group; it can be seen by relative displacement among the particles along the length of the bundle. Prismatic particles are well-developed crystal faces with low $(<10: 1)$ to moderate $(10: 1$ to $20: 1$ ) aspect ratios. They have one elongated dimension and two shorter of approximately equal dimensions (i.e., similar particle width and thickness, figure $6 \mathrm{~b}$ ). The sides of prismatic particles are 
Table 6. EPMA and structural formula of chlorite, from the garnet-cordierite-hypersthene-gedrite gneiss of the study area.

\begin{tabular}{|c|c|c|c|c|c|}
\hline \multirow[b]{2}{*}{ Domain } & \multicolumn{5}{|c|}{ Sample R-91-97 } \\
\hline & $96 / 1$ & $97 / 1$ & $98 / 1$ & $99 / 1$ & $100 / 1$ \\
\hline $\mathrm{SiO}_{2}$ & 44.11 & 45.05 & 40.36 & 39.91 & 41.67 \\
\hline $\mathrm{TiO}_{2}$ & 0 & 0 & 0 & 0 & 0 \\
\hline $\mathrm{Al}_{2} \mathrm{O}_{3}$ & 24.45 & 23.09 & 21.5 & 21.18 & 24.9 \\
\hline $\mathrm{Fe}_{2} \mathrm{O}_{3}$ & 13.08 & 12.20 & 15.94 & 15.84 & 12.98 \\
\hline $\mathrm{FeO}$ & 0 & 0 & 1.79 & 1.79 & 0 \\
\hline $\mathrm{MnO}$ & 0 & 0 & 0 & 0 & 0 \\
\hline $\mathrm{MgO}$ & 5.20 & 5.13 & 5.46 & 5.36 & 4.9 \\
\hline $\mathrm{CaO}$ & 1.14 & 1.11 & 1.04 & 1.06 & 1.16 \\
\hline $\mathrm{Na}_{2} \mathrm{O}$ & 0.18 & 0.35 & 0.11 & 0.1 & 0.2 \\
\hline $\mathrm{K}_{2} \mathrm{O}$ & 0.88 & 0.89 & 0.59 & 0.50 & 0.83 \\
\hline $\mathrm{BaO}$ & 0.38 & 0.24 & 0.17 & 0.34 & 0.28 \\
\hline $\mathrm{Cl}$ & 0.06 & 0.20 & 0.06 & 0.08 & 0.05 \\
\hline $\mathrm{H}_{2} \mathrm{O}^{*}$ & 12.92 & 12.74 & 12.14 & 11.98 & 12.52 \\
\hline Total & 102.41 & 101.01 & 99.17 & 98.15 & 99.50 \\
\hline $\mathrm{O}=\mathrm{F}, \mathrm{Cl}$ & 0.01 & 0.05 & 0.013 & 0.01 & 0.01 \\
\hline Total & 102.39 & 100.97 & 99.16 & 98.13 & 99.48 \\
\hline \multicolumn{6}{|c|}{28 oxygen } \\
\hline $\mathrm{Si}$ & 7.87 & 8.00 & 7.60 & 7.61 & 7.67 \\
\hline $\mathrm{Al}^{\mathrm{IV}}$ & 0.13 & 0 & 0.40 & 0.39 & 0.33 \\
\hline$\Sigma \mathrm{X}$ & 8 & 8 & 8 & 8 & 8 \\
\hline $\mathrm{Al}^{\mathrm{VI}}$ & 5.21 & 5.08 & 4.60 & 4.59 & 5.27 \\
\hline $\mathrm{Ti}$ & 0 & 0 & 0 & 0 & 0 \\
\hline $\mathrm{Fe}^{3+}$ & 1.76 & 1.65 & 2.26 & 2.27 & 1.80 \\
\hline $\mathrm{Fe}^{2+}$ & 0 & 0 & 0.28 & 0.29 & 0 \\
\hline Mn & 0 & 0 & 0 & 0 & 0 \\
\hline $\mathrm{Mg}$ & 1.38 & 1.37 & 1.53 & 1.52 & 1.34 \\
\hline $\mathrm{Ca}$ & 0.22 & 0.21 & 0.21 & 0.22 & 0.23 \\
\hline $\mathrm{Na}$ & 0.12 & 0.24 & 0.08 & 0.07 & 0.14 \\
\hline K & 0.40 & 0.41 & 0.28 & 0.24 & 0.39 \\
\hline $\mathrm{Ba}$ & 0.05 & 0.03 & 0.03 & 0.05 & 0.04 \\
\hline $\mathrm{Cl}$ & 0.04 & 0.12 & 0.04 & 0.05 & 0.03 \\
\hline $\mathrm{X}_{\mathrm{Mg}}$ & 0.44 & 0.45 & 0.38 & 0.37 & 0.43 \\
\hline
\end{tabular}

typically parallel. Prismatic particle's edges are well defined and crystalline in nature. Fibrous forms of gedrite are thin and elongated particles with parallel sides and their surfaces are smooth. Fibres have very high (20:1 to 100:1, or higher) aspect ratios and often display curvature (i.e., bending and flexibility (figure 6b) where the fibre end wraps around another particle). This classification of amphibole has been given by Harris et al. (2007). TEM image shows the different position occupied by gedrite and arrangement of double-chain silicate structure with all the three axial directions. Unit cell parameter of the gedrite detected by TEM are $a$-axis $=18.6 \AA$, $b$-axis $=17.8 \AA$ and $c$-axis $=5.58 \AA$. It is very similar to gedrite unit cell parameter, i.e., $a$-axis $=1.8531 \mathrm{~nm}, b$-axis $=1.7741 \mathrm{~nm}$ and $c$ axis $=0.5249 \mathrm{~nm}$; given by Papike and Rose (1970). The SAED pattern shows the distribution of metallic element position at the different lattice site.

\section{Metamorphic evolution}

\subsection{Phase compatibility relation}

The garnet-cordierite-gedrite-hypersthene gneisses are plotted in $\mathrm{FeO}-\mathrm{MgO}-\mathrm{Al}_{2} \mathrm{O}_{3}-\mathrm{SiO}_{2}-\mathrm{H}_{2} \mathrm{O}$ (FMASH) system in AFM projection diagram. Mineral parageneses that result from the reaction between garnet and gedrite reflect the stability of garnet-cordierite-gedrite join. The mineral association is a product of poly-stage metamorphism, i.e., it is an association of mineral assemblages of different metamorphic stages which are supported by the following metamorphic reactions:

Early stage: Prograde reaction:

$$
\text { chlorite }+ \text { quartz }=\text { garnet } 1+\text { gedrite } 1,
$$

$$
\text { Al-rich biotite }=\text { gedrite } 1+\text { cordierite } \text {. }
$$

Middle stage: Prograde reaction:

$$
\begin{aligned}
& \text { garnet } 1=\text { cordierite }+ \text { gedrite } 2, \\
& \text { garnet } 1=\text { gedrite } 2+\text { cordierite, }
\end{aligned}
$$

$$
\text { garnet } 1+\text { biotite }=\text { gedrite } 2+\text { cordierite } .
$$

Late stage:

gedrite $3=$ orthopyroxene + garnet $2+$ cordierite .

The three-phase field of garnet-gedrite-biotite from the AKF diagram suggests that the cordieritegarnet-biotite field was terminated with the formation of new assemblages of cordierite-gedritebiotite according to reaction (8). Reaction (8) in the given form has been studied at low pressure by Knabe (1970). According to Winkler (1976), biotite with higher $\mathrm{X}_{\mathrm{Mg}}$ that was used by Knabe (1970) would also produce cordierite at higher pressures. However, it is evident from the petrogenetic grid of Grant (1973), the assemblages 


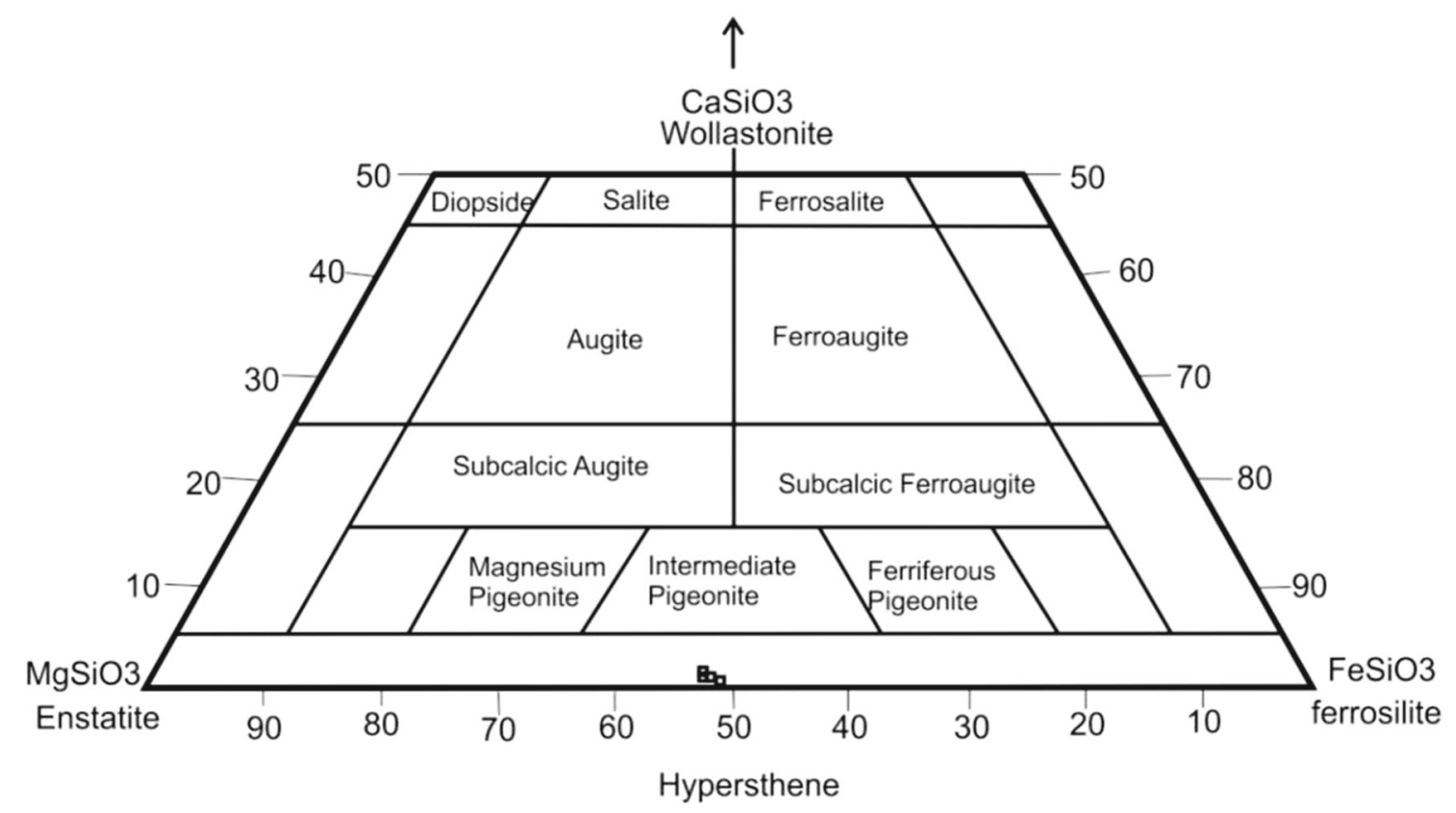

Figure 3. Plot of EPMA data of pyroxene shown in part of triangular diagram $\mathrm{CaSiO}_{3}-\mathrm{MgSiO}_{3}-\mathrm{FeSiO}_{3}$.

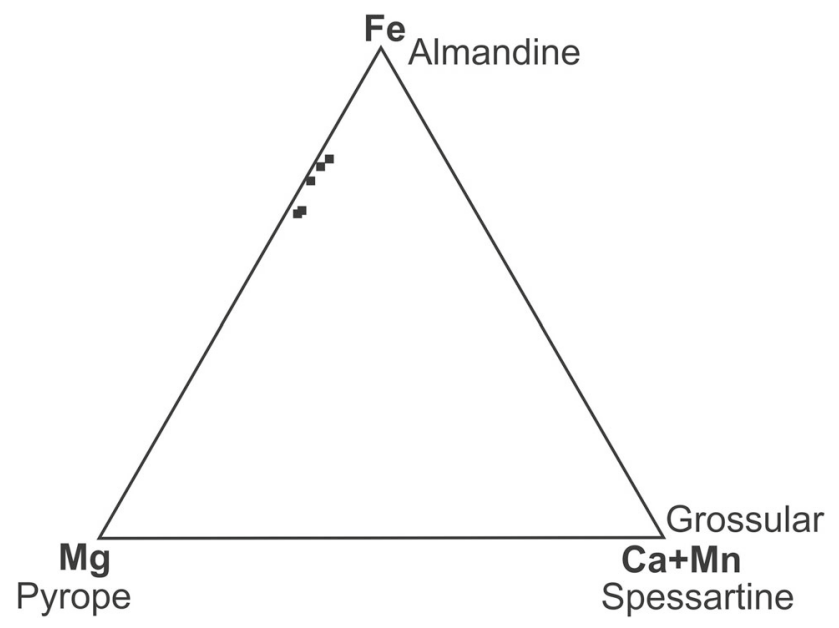

Figure 4. Plot showing the variation in spessartinegrossularite-almandine-pyrope endmember composition in the garnet.

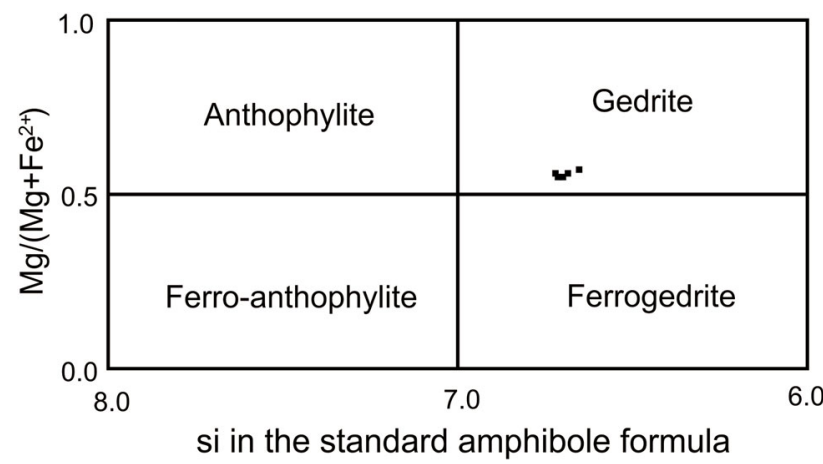

Figure 5. Leaks classification diagram for the amphiboles.
gedrite-cordierite-garnet-K-feldspar may be found in both high pressure, high temperature and relatively low pressure, low temperature of his diagram. In the three-phase field of gedriteorthopyroxene-garnet and gedrite-cordieritegarnet (figure 7a), orthopyroxene appears from reaction (12) and can be represented by the phase compatibility diagram change from figure 7 (a and b). The three stages of gedrite obtained from the TEM analyses are also confirmed by metamorphic evolution of the gedrite1 (elongated and defined the foliation in the gneiss) appear due to reactions (7) and (8) during the early stage, gedrite2 (idioblastic) formed due to reactions (9)-(11) during the middle stage and finally gedrite3 (prismatic) break down to form hypersthenes and garnet2 due to reaction (12) during the late stage of evolution of the gneisses.

\subsection{Petrogenetic grid}

A petrogenetic grid of the garnet-cordieritegedrite-hypersthene gneisses in FMASH system is shown in figure 8 . The topology in the divariant fields is depicted by the AFM diagram. Quartz, albite, K-feldspar and water are considered as excess phases. In four component system containing five phases, viz., garnet, gedrite, cordierite, biotite, orthopyroxene, and five univariant reactions will diverge from one invariant point. The slope of the dehydration reactions is calculated 

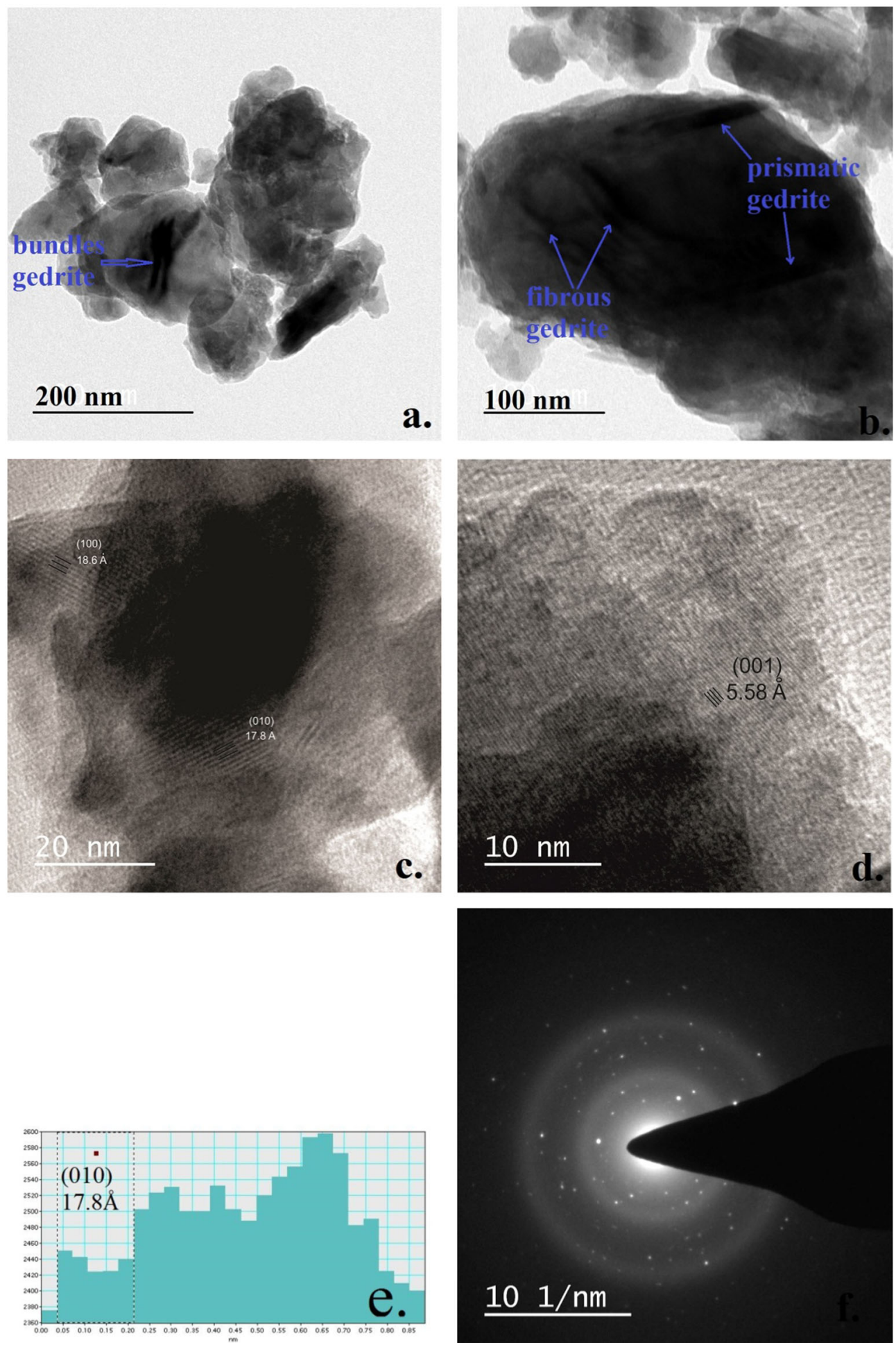

Figure 6. TEM images (a) shows the distribution of gedrite grain in which bundles form of gedrite grains are present. (b) Fibrous and prismatic growth of gedrite minerals. (c) The orientations of grains are in (100) and (010). (d) The orientation of grain in (001). (e) Width of double-chain silicate structure along (010) orientation with the help of histograph by using TEM. (f) SAED pattern of gedrite grain.

after the method proposed by Ferry and Burt (1982). Each univariant reaction is designated by a phase within bracket which does not participate in the reaction. Figure 8 depicts that the invariant point of the reactions is the thermal peak condition of the metamorphism in which the observed reactions (11) and (12) are involved as univariant reactions. 


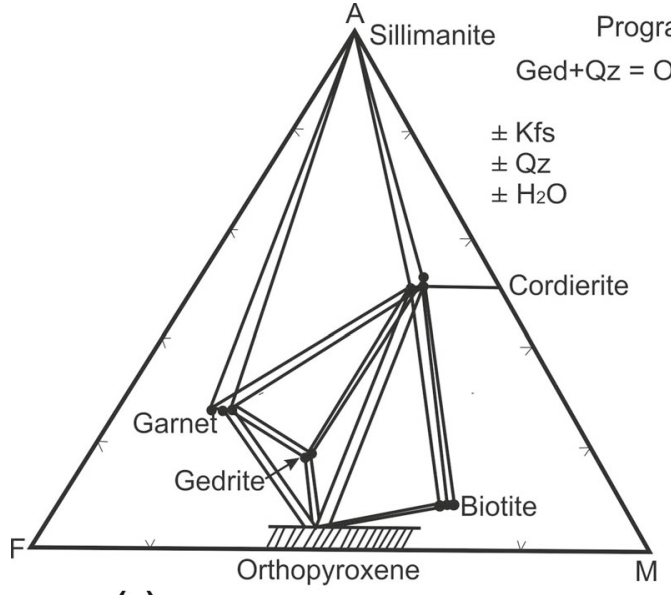

(a)

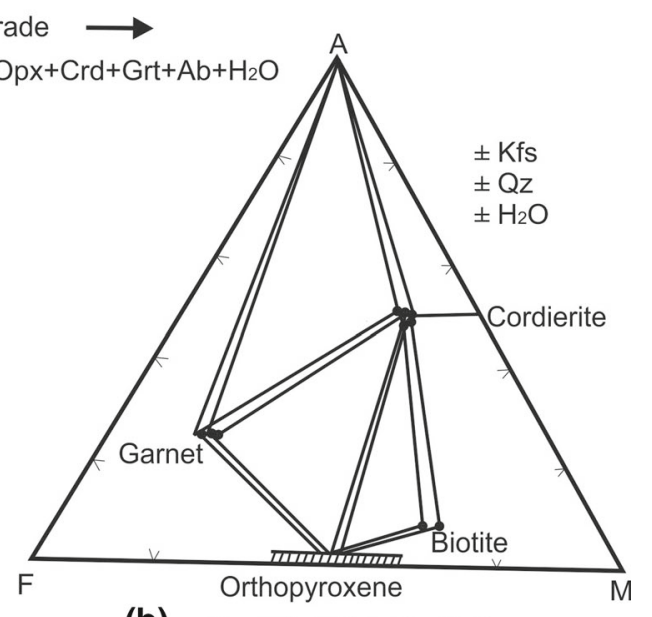

(b) $\mathrm{Opx}+\mathrm{Crd}+\mathrm{Grt}+\mathrm{Ab}+\mathrm{H}_{2} \mathrm{O}$

Figure 7. AFM projection from K-feldspar point of the AKFM tetrahedron onto the AFM plane: (a) showing the gedrite in the middle of triangle Grt-Crd-Opx in which gedrite is reactant; (b) depicts the disappearance of gedrite to form the triangle Grt-Crd-Opx as a product, where $\mathrm{A}=\mathrm{Al}_{2} \mathrm{O}_{3}+\mathrm{Fe}_{2} \mathrm{O}_{3}-\left(\mathrm{K}_{2} \mathrm{O}+\mathrm{Na}_{2} \mathrm{O}+\mathrm{CaO}\right) ; \mathrm{F}=\mathrm{FeO}+\mathrm{MnO} ; \mathrm{M}=\mathrm{MgO}$.

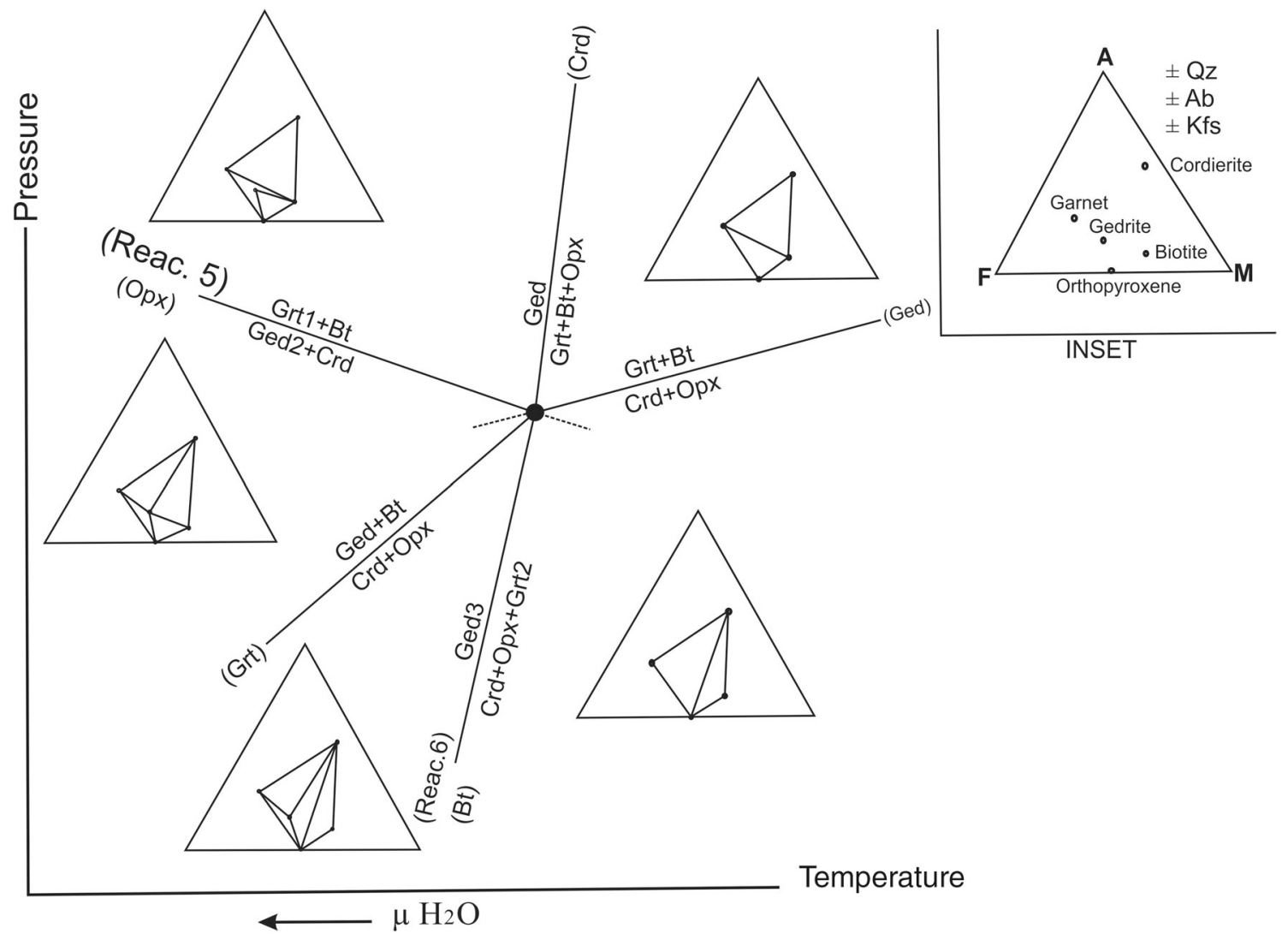

Figure 8. A petrogenetic grid in the FMASH system constructed after the Schreinmakers analysis for the garnet-cordieritehypersthene-gedrite gneiss.

\section{$4.3 P-T$ condition}

Accurately estimating the $P-T$ conditions is the most important work in understanding the thermal state of the past or the present-day lithospheric mantle. Naturally, the reliable $P-T$ estimates can only be obtained by applying the precise and accurate thermometers and barometers (Tam et al. 2012). The metamorphic rock of the area contains garnet-cordierite-gedrite gneiss and garnet-cordierite-gedrite-hypersthene gneiss. Their temperature and pressure conditions have been 
Table 7. Pressure and temperature estimates of the garnet-cordierite-hypesrsthene gniess of the study area through conventional geothermobarometers and internally consistent data set.

Estimate of geothermometers (temp. in ${ }^{\circ} \mathrm{C}$ ) at $7 \mathrm{kbar}$

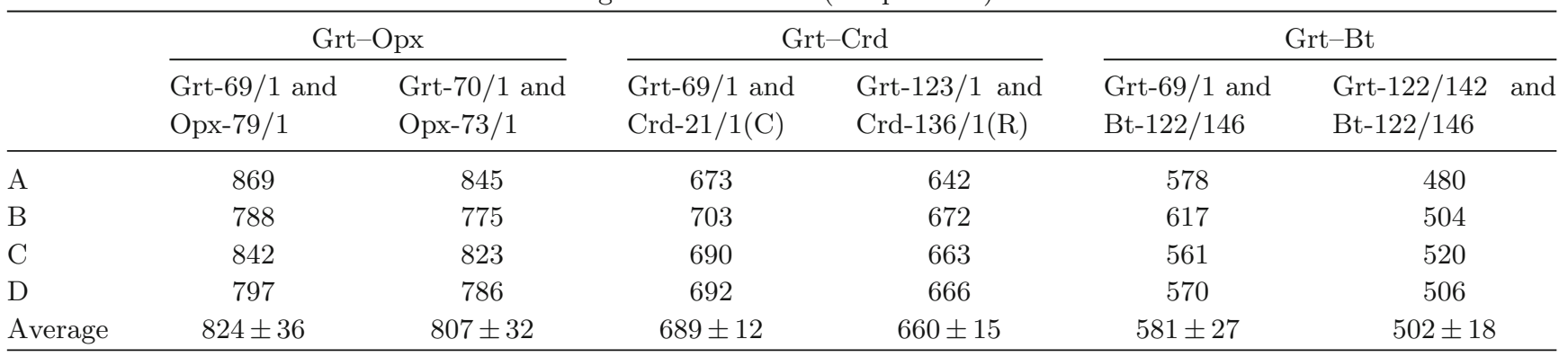

Estimate of geobarometers (pressure in kbar) average $P-T$ estimate

Garnet-cordierite-sillimanite-quartz geobarometer $E=595^{\circ} / 5.3 \mathrm{kbar}$

\begin{tabular}{ccc}
\hline & $\mathrm{P}-\mathrm{Mg}$ & $\mathrm{P}-\mathrm{Fe}$ \\
\hline $\mathrm{a}$ & 6.90 & 6.86 \\
$\mathrm{~b}$ & 7.79 & 6.76 \\
$\mathrm{c}$ & 7.60 & 7.08 \\
& $7.43 \pm 0.5$ & $6.9 \pm 0.16$
\end{tabular}

Note: In Grt-Opx, models of: A = Sen and Bhattacharya (1984); B = Bhattacharya et al. (1991); C = Lee and Ganguly (1988); D = Aranovich and Podlesskii (1989). In Grt-Crd: A = Thompson (1976); B = Bhattacharya et al. (1992); C = Dwivedi (1996); D = Dwivedi et al. (1998). In Grt-Bt: A = Thompson (1976); B = Dasgupta et al. (1991); C = Bhattacharya et al. (1992); D = Dwivedi et al. (2007); a = Thompson (1976); b = Wells (1979); c = Dwivedi et al. (1997); E= Thermocalc.

estimated by conventional garnet-orthopyroxene, garnet-biotite, garnet-cordierite exchange geothermometers and garnet-cordierite-sillimanitequartz geobarometers, as well as sets of independent reactions between end members of mineral, were calculated by THERMOCALC v3.1 (Powell and Holland 1988) using the internally consistent data set of Powell and Holland (1988). The estimated temperature with garnet-orthopyroxene geothermometry for garnet-cordierite-hypersthene gneiss by various models is given in table 7 . The temperature lies between $775^{\circ}$ and $869^{\circ} \mathrm{C}$ and the average temperature from the samples varies from $807^{\circ} \mathrm{C}$ to $824^{\circ} \mathrm{C}$. The average temperature obtained from different garnet-cordierite and garnet-biotite exchange geothermometers lies between $660-689^{\circ} \mathrm{C}$ and $502-581^{\circ} \mathrm{C}$, respectively. The average $P-T$ condition of metamorphism for the garnet-cordierite-hypersthene gneiss estimated from the intersection of the different set of metamorphic phases involving garnet, orthopyroxene, cordierite, biotite and gedrite (THERMOCALC v3.1 data set) is $595^{\circ} \mathrm{C} / 5.3 \mathrm{kbar}$. The pressure calculated for the garnet-cordieritegedrite-hypersthene gneiss with the help of garnet-cordierite-sillimanite-quartz $\mathrm{P}-\mathrm{Mg}$ and $\mathrm{P}-\mathrm{Fe}$ endmembers geobarometers varies from 6.76 to
$7.79 \mathrm{kbar}$ and the average pressure from $\mathrm{P}-\mathrm{Mg}$ and $\mathrm{P}-\mathrm{Fe}$ endmembers geobarometers lies between 6.9 and $7.43 \mathrm{kbar}$.

\section{Conclusions}

We first report the gedrite-bearing garnetcordierite-hypersthene gneiss, a high-grade granulite facies rock which occurs as enclaves within CGGC. The mineral association and textural relations suggest that the mineral assemblages were formed due to various prograde reactions (1-6) at different stages. The gedrite1 appears in the gneiss from the consumption of chlorite and Alrich biotite at $502-581^{\circ} \mathrm{C}$ temperature during the early stage and gedrite 2 was formed from the reaction garnet $1=$ cordierite + gedrite 2 , garnet 1 $=$ gedrite $2+$ cordierite and garnet $1+$ biotite $=$ gedrite $2+$ cordierite at $660-689^{\circ} \mathrm{C} / 6.76$ kbar during the middle stage. Hypersthene appears in the rocks due to the breakdown of the gedrite 3 due to the reaction gedrite $3=$ orthopyroxene + garnet $2+$ cordierite at thermal peak $869^{\circ} \mathrm{C} / 7.79 \mathrm{kbar}$ during the late stage. The three stages of gedrite have also been confirmed by the TEM analysis of the gneiss of the study area. Two generations of 
garnet are present in this rock, viz., garnet1 contains the low pyrope (20.9-26.4\%), and garnet2 includes the high pyrope (31.1-32.0\%) as compared to garnet1. The phase compatibility relationship of the mineral phases garnet, cordierite, gedrite and hypersthene and $P-T$ petrogenetic grid in FMASH system reveals the metamorphic evolution of the garnet-cordierite-hypersthene gneiss through the metamorphic reactions (1-6).

\section{Acknowledgements}

We are thankful to the Director, Indian Institute of Technology (BHU), for providing the facility to do the analytical works at the Central Instrumental Facility of the Institute. We appreciate the constructive comments of unanimous reviewer to improve the paper.

\section{References}

Aranovich L Ya and Podlesskii K K 1989 Geothermobarometry of high-grade metapelites: Simultaneously operating reactions; In: Evolution of metamorphic belts, Blakwell Scientific Publications, Oxford (eds) Cliff R A, Yardley B W D and Daly J S, Geol. Soc. Spec. Publ. 43 45-61.

Arnold J and Sandiford M 1990 Petrogenesis of cordierite orthoamphibole assemblages from the Springton region, South Australia; Contrib. Mineral. Petrol. 106 100-109.

Baidya T K, Maity N and Biswas P 1989 Tectonic phases and crustal evolution in a part of the eastern Chhotanagpur gneissic complex; J. Geol. Soc. India 34 318-324.

Baker J, Powell R and Sandiford M 1987 Corona textures between kyanite, garnet and gedrite in Gneisses from Errabiddly, western Australia; J. Metamorph. Geol. 55 357-370.

Bhattacharya A, Krishnakumar K R and RaithMandSen S K 1991 An improved set of $\mathrm{a}-\mathrm{x}$ parameter for $\mathrm{Fe}-\mathrm{Mg}$ $\mathrm{Ca}$ garnets and refinement of the orthopyroxene-garnet thermometer and the orthopyroxene-garnet-plagioclasequartz barometers; J. Petrol. 32 629-656.

Bhattacharya A, Mohanty L, Maji A, Sen S K and Raith M 1992 Non-ideal mixing in the phlogopite-annitebinary solution: Constraints from experimental data on $\mathrm{Mg}-\mathrm{Fe}$ partitioning and a reformulation of the biotite-garnet geothermometers; Contrib. Mineral. Petrol. 111 87-93.

Chatterjee N and Ghose N C 2011 Extensive early neoproterozoic high-grade metamorphism in north Chotanagpur gneissic complex of the central Indian tectonic zone; Gondwana Res. 20 362-379.

Dasgupta S, Sengupta P, Guha D and Fukuoka M 1991 A refined garnet-biotite $\mathrm{Fe}-\mathrm{Mg}$ exchange geothermometer and its application in amphibolites and granulites; Contrib. Mineral. Petrol. 109 130-137.

Dasgupta S, Sengupta P, Sengupta P, Ehl J and Raith M 1999 Petrology of gedrite-bearing rocks in mid-crustal ductile shear zones from the eastern Ghats Belt, India; J. Metamor. Geol. 17 765-778.
Dwivedi S B 1996 Non-ideal Mg-Fe binary mixing in cordierite: Constraints from experimental data on $\mathrm{Mg}-\mathrm{Fe}$ partitioning in garnet and cordierite and a reformulation of granet-cordierite geothermometer; J. Earth Syst. Sci. $105365-377$.

Dwivedi S B, Mohan A and Lal R K 1997 Internally consistent geothermobarometers in the system $\mathrm{FeO}$ $\mathrm{MgO}-\mathrm{Al}_{2} \mathrm{O}_{3}-\mathrm{SiO}_{2}-\mathrm{H}_{2} \mathrm{O}$ involving garnet, cordierite, aluminosilicate and quartz and their application to metapelites; J. Geol. Soc. India 49 647-660.

Dwivedi S B, Mohan A and Lal R K 1998 Recalibration of $\mathrm{Fe}-\mathrm{Mg}$ exchange reaction between garnet and cordierite as a thermometer; Eur. J. Mineral. 10 281-289.

Dwivedi S B, Jimmy L, Vanthanglana V and Lal S N 2007 A refined garnet-biotite thermometer: Constraints from empirically derived Margulus parameters for garnet $(\Delta \mathrm{WCa} \& \Delta \mathrm{WMn})$ and biotite $(\Delta \mathrm{WAl} \& \Delta \mathrm{WTi}) ;$ Ind. Mineral. 41 97-211.

Ferry J M and Burt D M 1982 Characterization of metamorphic fluid through mineral equilibria; In: Characterization of metamorphism through mineral equilibria (ed.) Ferry J M, Rev. Miner. 10 207-262.

Ghose N C 1983 Geology, tectonics and evolution of the Chhotanagpur granite gneiss complex, Eastern India; In: Structure and tectonics of precambrian rocks of India, recent researches in geology (ed.) Sinha-Roy S, Vol. 10, pp. 211-247.

Ghose N C 1992 Chhotanagpur gneiss-granulite complex eastern India: Present status and future prospect; Indian J. Geol. 84 100-121.

Goswami B and Bhattacharyya C 2010 Tectonothermal evolution of Chhotanagpur granite gneiss complex from northeastern part of Puruliya district, west Bengal, eastern India; Indian J. Geol. 80 41-54.

Grant J A 1973 Phase equilibria in high-grade metamorphism and partial melting of pelitic rocks; Am. J. Sci. $273289-317$.

Harris K E, Bunker K L, Strohmeier B R, Hoch R and Lee R J 2007 Discovering the true morphology of amphibole minerals: Complementary TEM and FESEM characterization of particles in mixed mineral dust; Mod. Res. Educ. Top. Microsc. 3(2) 643-650.

Karmakar S, Bose S, BasuSarbadhikari A and Das K 2011 Evolution of granulite enclaves and associated gneisses from Purulia, Chhotanagpur granite gneiss complex, India: Evidence for 990-940 Ma tectonothermal event(s) at the eastern India cratonic fringe zone; J. Asian Earth Sci. 4 69-88.

Knabe W 1970 Reaction der biotitebei der anatexis Geology; JB 88 355-372.

Lal R K, Ackermand D, Maraase P and Seifert F 1984 Sapphirine-bearing assemblages from kiranur, southern India. A study of chemo graphic relationships in $\mathrm{Na}_{2} \mathrm{O}$ FeO-MgO- $\mathrm{Al}_{2} \mathrm{O}_{3}-\mathrm{SiO}_{2}-\mathrm{H}_{2} \mathrm{O}$ system; N. Jahrb. Min. Abh. 150 121-152.

Leake B E, Woolley A R, Arps C E S, Birch W D, Gilbert M C, Grice J D, Howthorne F C, Kato A, Kisch H J, Krivovichev V G, Linthout K, Laird J, Mandarino J A, Maresch W V, Nickel E H, Rock N M S, Schumacher J C, Smith D C, Stephenson N C N, Ungaretti L, Whittaker E J W and Youzhi G 1997 Nomenclature of amphiboles: Report of the subcommittee on amphiboles of the 
international mineralogical association, commission on new minerals and mineral names; Can. Mineral. 35219 246.

Lee H Y and Ganguly J 1988 Equilibrium compositions of coexisting garnet and orthopyroxene: Experimental determinations in the system $\mathrm{FeO}-\mathrm{MgO}-\mathrm{Al}_{2} \mathrm{O}_{3}-\mathrm{SiO}_{2}$, and applications; J. Petrol. 29 93-113.

Mazumdar S K 1988 Crustal evolution of the Chhotanagpur gneissic complex and the Mica belt of Bihar; In: Pre Cambrian of the eastern Indian shield (ed.) Mukhopadhyay D, J. Geol. Soc. India 8 48-78.

Papike J J and Rose M 1970 Gedrite, crystal structures and intracrystallinecation distribution (abs); Am. Mineral. 55 304-305.

Peck W H and Smith M S 2005 Cordierite-gedrite rocks from the central metasedimentary belt boundary thrust zone (Grenville Province, Ontario): Mesoproterozoic metavolcanic rocks with affinities to the composite arc belt; $C a n$. J. Earth Sci. 42 1815-1828.

Powell R and Holland T J B 1988 An internally consistent dataset with uncertainties and correlations: 3. Applications to geobarometry, worked examples and a computer program; J. Meta. Geol. 6 173-204.

Robinson P, Spear F S, Schumacher J C, Laird J, Klein C, Evans B W and Doolan B L 1982 Phase relations in grid for the system $\mathrm{K}_{2} \mathrm{O}-\mathrm{FeO}-\mathrm{MgO}-\mathrm{Al}_{2} \mathrm{O}_{3}-\mathrm{SiO}_{2}-\mathrm{H}_{2} \mathrm{O}$ with the metamorphic amphiboles: Natural occurrence and theory particular reference to contact metamorphosedpelites (eds) Veblen D R and Ribbe P H, Mineral. Soc. Am., Rev. Mineral. 9B 1-227.

Sarkar A N 1988 Tectonic evolution of the Chhotanagpur plateau and the Gondwana basins in Eastern India: An interpretation based on supra-subduction geological processes; In: Precambrian of the eastern Indian shield (ed.) Mukhopadhyay D, Mem. Geol. Soc. India 8 127-146.

Sen S K and Bhattacharya A 1984 An orthopyroxene garnet thermometer and its application to the Madras Charnockites; Contrib. Mineral. Petrol. $\mathbf{8 8}$ $64-71$.

Corresponding editor: PulaK SEngupta
Sengupta D K and Sarkar S N 1964 Structure of the granitic rock and associated metamorphites of the area around Muri-Silli-Jhalida, Ranchi and Purulia Districts, India; In: Proceedings of the 22nd International Geological Congress, Vol. 4, pp. 374-389.

Sengupta D K and Sarkar S N 1968 Structure of granitic rocks and associated metamorphites of the area around Muri-Silli-Jhalida, Ranchi and Purulia Districts, India; Trans. Geol. Metall. Inst. India 65 1-18.

Sharma R S and Mac Rae N D 1981 Paragenetic relations in gedrite-cordierite-staurolite-biotite-sillimanitekyanite-gneisses at Ajitpura, Rajasthan, India; Contrib. Mineral. Petrol. 78 48-60.

Spear F S 1993 Metamorphism of ultramafic and cordieriteanthophyllite rocks; In: Metamorphic phase equilibria and pressure-temperature-time paths; Miner. Soc. Am. Monogr. Ser., Washington DC, pp. 393-489.

Srivastava S C and Ghose N C 1992 Petrology of the highgrade gneisses and granites around Chianki, south of Daltonganj, district Palamau Bihar; Indian J. Geol. 64 122-142.

Tam P Y, Zhao G, Sun M, Li S, Wu M and Yin C 2012 Petrology and metamorphic $\mathrm{P}-\mathrm{T}$ path of high-pressure mafic granulites from the Jiaobei massif in the Jiao-LiaoJi Belt, North China Craton; Lithos 155 94-109.

Thompson A B 1976 Mineral reaction in pelitic rocks: I. Prediction in $\mathrm{P}-\mathrm{T}-\mathrm{X}(\mathrm{Fe}-\mathrm{Mg})$ phase relations. II. Calculations of some $\mathrm{P}-\mathrm{T}-\mathrm{X}(\mathrm{Fe}-\mathrm{Mg})$ phase relations; $\mathrm{Am}$. J. Sci. 276 401-454.

Wells P R A 1979 Chemical and thermal evolution of Archean sialic crust, Southern West Greenland; J. Petrol. 20 187-226.

Whitney D L and Evans B W 2010 Abbreviations for names of rock-forming minerals; Am. Miner. 95 185-187.

Winkler H G F 1976 Petrogenesis of metamorphic rocks; 4th edn, Springer, New York.

Yadav B S, Wanjari N, Ahmad T and Chaturvedi R 2016 Geochemistry and petrogenesis of Proterozoic granitic rocks from northern margin of the Chotanagpur Gneissic Complex (CGC); J. Earth Syst. Sci. 125 1041-1060. 